\title{
CONCILIACIÓN Y ACTIVIDAD FÍSICA EN FAMILIAS CON HIJOS EN ENSEÑANZA SECUNDARIA OBLIGATORIA (12-16 AÑOS)
}

\author{
WORK-LIFE BALANCE AND PHYSICAL ACTIVITY IN FAMILIES WITH CHILDREN IN \\ SECONDARY EDUCATION (12-16 YEARS)
}

\author{
Raúl Fraguela-Vale \\ Lara Varela-Garrote ${ }^{2}$ \\ Juanjo Lorenzo-Castiñeiras ${ }^{3}$ \\ Tania Merelas-Iglesias ${ }^{4}$
}

Resumen

La conciliación de la vida laboral, familiar y personal hace referencia a las posibilidades de compaginar y armonizar los tiempos de trabajo con las actividades familiares y personales de los ciudadanos. Los objetivos del presente trabajo son conocer los niveles de actividad física de las familias con hijos en Enseñanza Secundaria Obligatoria (ESO) -12 a 16 años- y analizar la relación de la conciliación de la vida laboral, familiar y personal de los progenitores con los siguientes factores: a) actividad física de los adultos y sus hijos y b) actividad física compartida en familia. Una muestra de 859 familias gallegas con hijos en la Enseñanza Secundaria Obligatoria participaron en el estudio. Dentro del grupo familiar, uno de los padres debía completar un cuestionario en el que manifestaba sus niveles de conciliación, de práctica de actividad física y de la de sus hijos. Los resultados indican que los niveles de actividad física de las familias (tanto de los adultos como de los adolescentes) son moderados. La conciliación es un factor relevante en la práctica de actividad física de los progenitores y en la práctica motriz compartida en familia. La práctica de los hijos no se encuentra relacionada con los niveles de conciliación de sus padres.

Palabras claves: conciliación; actividad física; familia; adolescentes

Abstract

Work-life balance is linked to the possibilities citizens have to combine and harmonize work schedules with family and personal activities. The aims of this work are to know the levels of physical activity in families with children in Secondary Education (12-16 years) and to analyze the relationship between parents' work-life balance with the following factors: a) parents' and children's physical activity, and b) shared family physical activity. A sample of 859 Galician families with children in Secondary Education participated in the study. One of the parents had to answer a questionnaire to report his levels of work-life balance and physical activity practice and the physical activity levels of his children. Findings show that family physical activity levels (both for adults and adolescents) are moderate. Work-life balance is a key factor in parents' physical activity and shared family physical activity. Adolescents' physical activity is not related with parents' work-life balance.

Keywords: work-life balance; physical activity; family; adolescents

Fecha de recepción: 20 de octubre de 2015

Fecha de aprobación: 15 de marzo de 2016

Para citar este artículo:

Fraguela-Vale, R., Varela-Garrote, L., Lorenzo-Castiñeiras, J. Merelas-Iglesias, T. (2016). Conciliación y actividad física en familias con hijos en Enseñanza Secundaria Obligatoria (12-16 años). Lúdica Pedagógica, (23), 43-51.

1 Licenciado y doctor en Educación Física. Profesor titular de Escuela Universitaria en la Universidad de la Coruña. Correo electrónico: raul.fraguela@udc.es

2 Licenciada y doctora en Educación Física. Profesora contratada doctora en el Departamento de Didácticas Específicas (área de Expresión Corporal) de la Universidad de la Coruña. Correo electrónico: lara@udc.es

3 Licenciado en Sociología. Investigador en Fundación Meniños y en la Universidad de Santiago de Compostela. Correo electrónico: juanjo.lorenzo@usc.es

4 Diplomada en Educación Social y licenciada en Pedagogía. Universidad de Santiago de Compostela. Correo electrónico: tania.merelas@usc.es 


\section{INTRODUCCIÓN}

Este estudio toma como referencia dos aproximaciones teóricas fundamentales en el binomio actividad físicafamilia: la transmisión de conductas saludables a los hijos y la práctica compartida de actividad física.

El interés por estudiar el hábito de actividad físicodeportiva guarda relación con los diversos beneficios físicos y emocionales que se derivan de su práctica (Codina y Pestana, 2012). En este sentido, destacamos la importancia de conocer la influencia que ejercen los entornos familiares en los hábitos de vida de los hijos, especialmente en edades tempranas. La práctica de actividad física es una de las dimensiones del comportamiento de la infancia en la que se ha detectado un fuerte influjo de la familia, que tiende a debilitarse a medida que aumenta la edad.

Igualmente, cabe señalar que hay una tendencia que apunta hacia un aumento de la probabilidad de adherencia y continuidad a la actividad física en edades tempranas si la familia posee un estilo de vida activo (Mulhall, Reis, y Begum, 2011) y existe apoyo social de los padres hacia la práctica motriz de sus hijos (Salazar, Feu, Vizuete Carrisoza, y De la Cruz-Sánchez, 2013). Sin embargo, se encuentran variaciones importantes en los resultados según factores como el tipo de familia, el estatus socioeconómico o la edad de los hijos. Sobre este último aspecto, Valdemoros, Ponce de León, Ramos y Sanz (2011) estudiaron una muestra de escolares de Educación Primaria y Secundaria, y hallaron que la influencia de los progenitores era mayor en el primer caso, mientras que se reducía en la adolescencia, a favor del grupo de iguales. El incremento de la autonomía, la influencia de los amigos y el cambio en las preferencias de práctica física, entre otros factores, provocan una reducción de la influencia que ejercen los progenitores en relación a la práctica de actividad física de sus hijos. En esta línea, García-Moya, Moreno, Rivera, y Jiménez-Iglesias (2011) evidencian la influencia que el grupo de iguales ejerce en la motivación para la práctica físico-deportiva; un proceso con especial repercusión durante la adolescencia, momento clave en los procesos de abandono deportivo (Cecchini, Méndez, y Contreras, 2005; Isorna, Ruiz, y Rial, 2013).

La conciliación de la vida laboral, familiar y personal (en adelante conciliación) es un factor global que ha ido adquiriendo relevancia en el análisis de la práctica de actividad física. En este estudio se plantea la conciliación como un fenómeno social, personal y laboral de gran calado (Borràs, Torns, y Moreno, 2007) que afecta a la familia y a sus responsabilidades, se asume tal conciliación como la relación de los tiempos de trabajo y la posibilidad de compaginarlos con la variedad de actividades personales (Canet, Grisolía, y Querol, 2006).

Inicialmente se incorporó al estudio de sectores con especiales dificultades en la organización de sus tiempos, como las familias con hijos pequeños (Rueda, Artazcoz, y Cortés, 2009), monoparentales (Tobío y Fernández, 1999) o las mujeres con trabajo remunerado (Martínez, Martín, Del Hierro, Jiménez-Beatty, y González, 2008), pero ha terminado por generalizarse al resto de la población. El estudio de la conciliación aporta información sobre cómo afecta la organización de los tiempos de las familias a la práctica de actividad física de sus integrantes. Un ejemplo del impacto del conflicto trabajo-familia en la práctica de actividad física lo proporciona el estudio de Root y Wooten (2008), en el que identificaron que las familias cuyos progenitores trabajan a turnos, especialmente si son de tarde, tienen dificultades para implicarse en la práctica deportiva organizada de sus hijos, lo cual puede provocar que la infancia no acuda a este tipo de actividades con la frecuencia deseada.

Si una mejor conciliación se asocia con una mayor práctica motriz de los adultos y esta última favorece que sus hijos practiquen, la conciliación podría tener efecto sobre los niveles de actividad de los adolescentes. Sin embargo, esta relación no se ha estudiado suficientemente. Todavía no se ha podido determinar en qué medida la organización de los tiempos adultos afecta a los niveles de práctica de los adolescentes.

Otro elemento relevante en el estilo de vida activo de las familias que puede verse afectado por la conciliación de los adultos es la actividad física compartida. Es interesante determinar hasta qué punto las limitaciones temporales condicionan los momentos y espacios para la práctica conjunta de los adultos y sus hijos. También si esta modalidad de práctica motriz tiene un efecto relevante sobre los niveles de actividad física cotidiana de ambos colectivos.

Los beneficios de la práctica compartida en familia no se limitan a la ejercitación física. En un estudio realizado con madres e hijas sedentarias que participaron con- 
juntamente en un programa de actividad física, además de incrementar sus niveles de práctica motriz, ambos grupos informaron de una mejora en la relación con sus madres/hijas como consecuencia de compartir esta experiencia (Ransdell et al., 2003). Estos beneficios se han documentado especialmente en aquellos contextos socioculturales en los que la participación en actividades físicas y deportivas por parte de la mujer cuenta con una menor tradición y aceptación social (Kargarfard et al., 2012). En estos casos, la implicación de las madres actúa como estímulo y legitimación de la práctica motriz de sus hijas. Sin embargo, la posibilidad de ejercitarse juntos no siempre depende de la voluntad de las familias, por lo que en este trabajo se estudia si las dificultades para organizar sus tiempos limitan la posibilidad familiar de realizar actividad física conjuntamente.

En un estudio previo realizado en familias con hijos en Educación Primaria (Fraguela, Castiñeiras y Varela, 2011), las personas con mayores niveles de conciliación eran más activas, lo que repercutía en la actividad de sus hijos. Una de las futuras líneas de investigación propuestas en el citado trabajo era ampliar el estudio a otras etapas educativas. Se asume aquí esa propuesta, que concretamos en los siguientes objetivos: 1) conocer los niveles de actividad física de las familias con hijos en Enseñanza Secundaria Obligatoria (ESO), 2) analizar la relación existente entre la conciliación y los siguientes factores: a) práctica de actividad física de los progenitores y de sus hijos adolescentes y b) actividad física compartida en familia.

\section{MÉTODO}

\section{Participantes}

Para la selección de la muestra se aplicó un muestreo aleatorio estratificado, por provincia, titularidad del centro (público, concertado o privado) y ciclo educativo (1.o o 2.ํㅜㅇco de la ESo). De las 1111 familias gallegas invitadas a participar en el estudio en 16 centros de ESO, finalmente fueron $859(77,31 \%)$ las que configuraron la muestra. Se trata de una participación elevada para la población de referencia: durante el curso 2010-2011, el alumnado matriculado en esta etapa educativa en Galicia fue de 88 016, según datos del Ministerio de Educación, Cultura y Deporte (2012). La muestra es representativa de esta población, con un error reducido ( $e=3,4 \%) \mathrm{y}$ un nivel de confianza adecuado (95,5\%). En la tabla 1 se resumen las características de la muestra.

Tabla 1. Datos de identificación de la muestra (\%)

\begin{tabular}{|c|c|c|c|c|c|c|c|}
\hline \multicolumn{2}{|c|}{ Quién responde } & \multicolumn{2}{|c|}{ Nivel de estudios } & \multicolumn{2}{|c|}{ Ingresos familiares (€) } & \multicolumn{2}{|c|}{ Titularidad del centro } \\
\hline Madre & 80,7 & \multirow{3}{*}{$\begin{array}{c}\text { Sin estudios } \\
\text { Primarios } \\
\text { Bachillerato }\end{array}$} & \multirow{2}{*}{$\begin{array}{c}0,8 \\
27,3\end{array}$} & \multirow{4}{*}{$\begin{array}{l}\text { - de } 1000 \\
1001-1500 \\
1501-2000 \\
2001-3000\end{array}$} & \multirow{3}{*}{$\begin{array}{c}9,0 \\
21,0 \\
25,4\end{array}$} & Público & 63,3 \\
\hline Padre & 19,3 & & & & & Concertado & 30,5 \\
\hline & & & 175 & & & Privado & 6,2 \\
\hline \multicolumn{2}{|c|}{ Sexo hijo } & \multirow{3}{*}{$\begin{array}{c}\text { FP } \\
\text { Superiores }\end{array}$} & \multirow{3}{*}{$\begin{array}{l}24,1 \\
29,6\end{array}$} & & 23,3 & \multicolumn{2}{|c|}{ Ciclo ESO } \\
\hline Chica & 53,6 & & & $3001-4000$ & 10,2 & $1 . \mathrm{o}$ & 59,2 \\
\hline Chico & 46,4 & & & + de 4000 & 11,1 & $2 . \mathrm{o}$ & 40,8 \\
\hline
\end{tabular}

Fuente: Elaboración propia.

\section{Instrumentos}

El cuestionario ha sido la técnica fundamental para la obtención de los datos que presentamos; es posible destacar cuatro bloques de análisis general en los que centramos el interés de este trabajo:
Nivel de práctica de actividad física: Se aplicó el índice de actividad física elaborado por Fraguela, Castiñeiras y Varela (2011) a partir de la frecuencia semanal con la que las personas pasean y realizan deporte. Sus valores oscilan entre 0 (no pasear ni hacer deporte ningún día a la semana) y 6 (pasear y hacer deporte más de cinco veces por semana cada actividad). 
Índice de conciliación: Se aplicó la "Escala de Conciliación de la Vida Laboral, Familiar y Personal" (Fraguela, Castiñeiras y Varela, 2011). Para facilitar el análisis e interpretación de los datos, se agruparon los valores de este índice en tres categorías: baja (valores entre 1,00 y 2,00 ), media (entre 2,01 y 3,00) y alta conciliación (entre 3,01 y 4,00 ).

Actividad física en familia: Se indica la persona o personas que realizan actividad física junto con sus hijos (la madre, el padre, ambos u otras personas).

\section{Procedimiento}

Los adolescentes recogieron el cuestionario en su centro educativo y tras recibir instrucciones de los investigadores en el propio colegio, se los entregaron a sus familias. Uno de los padres tenía que cumplimentar un cuestionario y manifestar en este su criterio con respecto a esta decisión, además de aportar la información solicitada en el cuestionario. Entre otros aspectos, el padre o madre de familia informó de sus niveles de conciliación, de su práctica de actividad física y de la de sus hijos. Se dio una semana de margen para la cumplimentación del instrumento. Transcurrido este plazo, los alumnos lo llevaron de vuelta al centro, el proceso terminó con la recogida de los cuestionarios por parte de los investigadores en el centro educativo.

\section{Análisis estadístico}

Se realizó un análisis descriptivo a través de análisis de frecuencias, medidas de tendencia central (media) y de dispersión (desviación típica) así como inferencial, mediante las pruebas Chi-cuadrado de Pearson, T de Student para dos muestras relacionadas, ANOVA (Analysis of variance) y ANCOVA (Analysis of covariance), y en los dos últimos casos a través del análisis post hoc de Tukey, cuando se encontraron diferencias significativas. Se trabajó con un nivel de confianza del $95 \%$. El análisis se realizó con el software sPSS versión 21.0.

\section{RESULTADOS}

Práctica de actividad física de las familias con hijos en ESO

La tabla 2 muestra la frecuencia de paseo y práctica deportiva de los miembros de la familia, así como su índice de actividad física. En el caso de los adultos, este último depende en gran medida del paseo y no tanto de la práctica deportiva. Para los adolescentes, ambas actividades contribuyen de forma similar al índice. Se confirma que los niveles de actividad de los progenitores son bajos: el índice oscila entre 0 y 6 puntos y sus valores medios se sitúan en torno a 2 . Sus hijos son significativamente más activos ( $\mathrm{T}$ Student para 2 muestras relacionadas: $t=-12,690 ; p=0,000)$, a pesar de lo cual su práctica motriz es moderada, al no alcanzar la mitad de la valoración máxima del índice.

Tabla 2. Paseo, práctica deportiva e índice de actividad física

\begin{tabular}{|l|l|c|c|c|c|c|}
\hline \multicolumn{2}{|c|}{} & N & Mín & Máx & & DT \\
\hline $\begin{array}{l}\text { Frecuencia con } \\
\text { la que pasea } \\
\text { o camina }\end{array}$ & $\begin{array}{l}\text { Usted } \\
\text { huijo/a }\end{array}$ & 833 & 0 & 3 & 1,59 & 0,97 \\
\cline { 2 - 7 } & Usted & 820 & 0 & 3 & 0,57 & 0,83 \\
\cline { 2 - 8 } $\begin{array}{l}\text { Frecuencia } \\
\text { con la que } \\
\text { hace deporte }\end{array}$ & $\begin{array}{l}\text { Su } \\
\text { hijo/a }\end{array}$ & 815 & 0 & 3 & 1,46 & 0,94 \\
\hline \multirow{2}{*}{$\begin{array}{l}\text { Índice de acti- } \\
\text { vidad física }\end{array}$} & Usted & 814 & 0 & 6 & 2,15 & 1,42 \\
\cline { 2 - 8 } & $\begin{array}{l}\text { Su } \\
\text { hijo/a }\end{array}$ & 807 & 0 & 6 & 2,97 & 1,55 \\
\hline
\end{tabular}

Fuente: Elaboración propia.

Respecto a la relación entre práctica de actividad física adulta y adolescente, el coeficiente de Pearson indica que existe una correlación positiva y altamente significativa entre los niveles de práctica de los progenitores y la de sus hijos $(r=0,258, p=0,000)$.

\section{Conciliación y práctica de actividad física}

En este punto se estudia si la conciliación (baja, media o alta) afecta a la práctica de actividad física de ocio de las familias con hijos adolescentes. El análisis de la varianza (tabla 3) indica que existen diferencias significativas en la práctica de actividad física según la conciliación de las familias. En el caso de los adultos, el análisis post hoc de Tukey identifica dos subconjuntos homogéneos: los que tienen niveles de conciliación bajos y medios, con índices de actividad física más bajos; y quienes se encuentran en la categoría "alta conciliación", que son más activos que los demás. 
Tabla 3. Práctica de actividad física en base al nivel de conciliación

\begin{tabular}{|l|c|c|c|c|c|c|}
\hline \multirow{2}{*}{$\begin{array}{c}\text { Actividad } \\
\text { Física }\end{array}$} & \multirow{2}{*}{$N$} & \multicolumn{3}{|c|}{$\begin{array}{c}\text { Conciliación } \\
\bar{x}(D T)\end{array}$} \\
\cline { 3 - 7 } & & Baja & Media & Alta & $g l$ & $F$ \\
\hline Usted & 1594 & $1,87(1,33)$ & $2,00(1,40)$ & $2,45(1,42)$ & 2 & $11,676^{* * *}$ \\
\hline Hijo/a & 1577 & $2,70(1,61)$ & $2,93(1,55)$ & $3,11(1,51)$ & 2 & $3,263^{*}$ \\
\hline
\end{tabular}

$* \mathrm{p}<0,05$

$* * * \mathrm{p}=0,000$

Fuente: Elaboración propia.

También se detectaron diferencias significativas en la práctica motriz adolescente en función de los niveles de conciliación de sus progenitores. Sin embargo, este dato se debe tomar con cautela, debido a la relación existente entre la conciliación y la actividad motriz de los adultos y de esta última con la de sus hijos. Es aconsejable, por tanto, realizar un análisis de la covarianza, estudiando el efecto de la conciliación sobre la actividad de los adolescentes, tomando como covariable la práctica de actividad física de los adultos. Los resultados de la prueba ANCova $(F=2,088, p>0,05)$ contradicen los encontrados en el análisis ANOVA, por lo que las variaciones encontradas en los niveles de práctica de actividad física de los adolescentes deben atribuirse a la actividad física que realizan sus padres (covariable) y no a sus niveles de conciliación (variable independiente).

\section{Conciliación y actividad física compartida en familia}

En este apartado se estudió la relación existente entre la conciliación y la práctica de actividad motriz junto con los hijos. Posteriormente, se analizó si esta última variable tiene incidencia sobre el índice de actividad física adulta y adolescente.
Respecto a la conciliación, a través del análisis Chicuadrado se detecta una asociación con la variable quién practica actividad física junto con mi hijo/a $\left(x^{2}=13,012, p<0,05\right)$. En las familias con bajos niveles de conciliación es poco probable que ambos progenitores realicen simultáneamente actividad física con sus hijos y es probable que ninguno de los dos lo haga y sí otras personas (familiares, amigos de los hijos, etc.). En las familias que concilian bien sus tiempos, la tendencia es la contraria. En todas las demás categorías (conciliación media y acompañamiento de un solo adulto a la práctica motriz del hijo), la asociación de ambas variables es débil.

Una vez comprobada la asociación entre la conciliación y la práctica física compartida en familia, se estudia si esta última incide sobre los niveles de actividad motriz, tanto de los adultos como de los adolescentes. El análisis de la varianza (tabla 4) indica que existen diferencias altamente significativas en los niveles de actividad física de los progenitores, según quién realiza ejercicio junto con sus hijos. Sin embargo, los niveles de práctica de los hijos no se ven afectados por esta variable.

Tabla 4. Práctica de actividad física en función de quién acompaña a los adolescentes

\begin{tabular}{|l|c|c|c|c|c|c|c|}
\hline \multirow{2}{*}{\begin{tabular}{c}
\multirow{2}{*}{$\begin{array}{c}\text { Actividad } \\
\text { física }\end{array}$} \\
\cline { 3 - 8 }
\end{tabular}} & \multirow{2}{*}{$N$} & \multicolumn{4}{|c|}{ Quién practica actividad física junto con mi hijo/a } & \multicolumn{2}{c|}{ Anova } \\
\cline { 3 - 8 } & & Usted & Su pareja & Ambos & Otros & $g l$ & $F$ \\
\hline Usted & 702 & $2,40(1,47)$ & $1,81(1,31)$ & $2,48(1,39)$ & $1,60(1,35)$ & 3 & $14,478^{* * *}$ \\
\hline Hijo/a & 700 & $2,99(1,50)$ & $2,93(1,62)$ & $3,18(1,42)$ & $3,07(1,61)$ & 3 & 1,056 \\
\hline
\end{tabular}

$* * * p=0,001$

Fuente: Elaboración propia. 
Para estudiar la naturaleza de las diferencias en los niveles de actividad física de los adultos, se recurre a las comparaciones múltiples por el procedimiento de Tukey (tabla 5). Se identifican dos subconjuntos homogéneos.

Tabla 5. Comparaciones post hoc (Tukey)

\begin{tabular}{|l|c|c|c|}
\hline \multirow{2}{*}{$\begin{array}{c}\text { Quien practica } \\
\text { actividad física } \\
\text { junto con mi hijo/a }\end{array}$} & \multirow{2}{*}{$\mathrm{N}$} & \multicolumn{2}{|c|}{ Índice de AF progenitores } \\
\cline { 3 - 4 } & & 1 & 2 \\
\hline & 103 & 1,6019 & \\
\hline Otras personas & 111 & 1,8108 & \\
\hline Mi pareja & 190 & & 2,4000 \\
\hline Yo mismo/a & 298 & & 2,4866 \\
\hline Ambos & & 0,579 & 0,952 \\
\hline Sig. & & & \\
\hline
\end{tabular}

Fuente: Elaboración propia.

Las personas que no realizan ejercicio conjuntamente con sus hijos, porque es su pareja u otras personas las que lo hacen, son menos activas. En el segundo subconjunto están aquellos adultos que acompañan a sus hijos a la práctica deportiva (bien solos o con sus parejas), que son significativamente más activos que los que no lo hacen.

\section{DISCUSIÓN}

Respecto a la práctica de actividad física de las familias con hijos en ESO, los resultados encontrados refuerzan la tendencia que informa de bajos niveles de práctica de actividad física en nuestro país, tanto en la población en general (Consejo Superior de Deportes, 2010; García, 2006), como entre adolescentes (Beltrán-Carrillo, DevísDevís, y Peiró-Velert, 2012).

El paseo es la actividad determinante en la práctica física adulta, muy por encima de la práctica deportiva. Estos resultados coinciden con los estudios que señalan que el paseo es un hábito de ocio extendido en España (Instituto Nacional de Estadística, INE, 2010), con una presencia mucho más importante que en el resto de la Unión Europea (Eurostat, 2007). También en el caso de la adolescencia, la actividad de caminar o pasear forma parte importante de la actividad física cotidiana, al mismo nivel que la práctica deportiva. Esta realidad probablemente esté ligada al acompañamiento durante el paseo de uno o ambos progenitores y al desplazamiento con el grupo de iguales, de creciente influencia en estas edades.

Se confirma la existencia de una relación positiva entre la práctica de actividad física de los progenitores y la de sus hijos, tendencia que ya había sido detectada en estudios previos (Wagner et al., 2004). La familia posee una cultura común a todos sus miembros que le otorga sentido a todo lo que se hace en el grupo. Esta cultura se corresponde con una serie de valores, normas, pautas y principios que dentro del seno familiar dictan la conducta y unen a sus miembros bajo una esfera de interpretación de la realidad que hace que externalicen prácticas similares (Crespo, 2011). La actividad física forma parte de esas prácticas, que no se asemejan necesariamente en la tipología (ya que las preferencias de padres e hijos no tienen por qué coincidir), pero sí en lo referente al gusto por el movimiento y las dinámicas activas en la esfera laboral-académica, comunitaria, recreativa y relacionada con el transporte. Estas tendencias, especialmente en el campo de estudio de la familia y la actividad física, suelen agruparse bajo la denominación de estilos de vida activos y su transmisión posee un marcado carácter cultural e intergeneracional.

Los resultados sobre conciliación y práctica de actividad física indican que mayores niveles de conciliación están asociados con índices más altos de actividad física en los adultos. Esta relación refuerza la tendencia apuntada por Moraleda (2006), que indica que las dificultades para compaginar la vida laboral y familiar, especialmente en el caso de las mujeres, suelen superarse disminuyendo el tiempo personal dedicado al ejercicio físico. En la misma línea, Hicks, Basu y Sappey (2011) encontraron que las obligaciones laborales y familiares eran la principal causa por la que los adultos perdieron algunas de sus sesiones semanales de actividad deportiva organizada.

Sin embargo, la conciliación de los progenitores no parece ejercer una influencia directa sobre la práctica de actividad física de los adolescentes. Este dato resulta relevante para comprender la naturaleza de su práctica motriz: los hábitos familiares influyen en sus niveles de actividad, pero no dependen de la disponibilidad de los adultos para acceder a esta práctica, ya que su nivel de autonomía les permite ejercitarse solos o con sus amigos. 
Las medidas destinadas a mejorar la conciliación de estas familias tendrían un efecto positivo sobre los niveles de actividad física de los adultos, lo que podría favorecer que la adolescencia fuese más activa. Sin embargo, no se trata de una relación directa: una mejor conciliación genera un potencial para que aquellas personas interesadas en realizar ejercicio puedan hacerlo. Si esto se convierte en un hábito y más aún, en un hábito de ambos progenitores, es más probable que sus hijos adolescentes sean activos. No cabe esperar que la simple disponibilidad de tiempo de los adultos genere este efecto entre los hijos.

Los datos obtenidos respecto a conciliación y actividad física compartida en familia permiten identificar tres modelos de práctica familiar. En el primero de ellos la conciliación no es determinante y consiste en que uno de los dos progenitores realiza ejercicio junto con su hijo. El segundo modelo está formado por las familias con bajos niveles de conciliación, en el que ninguno de los progenitores practica actividad física con su hijo, que se ejercita con otro familiar o con sus amigos. El tercer modelo está compuesto por familias con una conciliación alta, en las que ambos progenitores realizan actividad motriz junto con los adolescentes.

Estos tres modelos tienen una repercusión relevante sobre los niveles de actividad de los adultos, pero no de los adolescentes. Quienes acompañan a sus hijos en la práctica de actividad física son físicamente más activos y los niveles más altos de práctica motriz de los progenitores se dan cuando ambos adultos practican junto a sus hijos. Los progenitores que no practican junto a sus hijos ven reducidas sus posibilidades de realizar actividad física. Sin embargo, esta relación no se cumple para los adolescentes: su nivel de actividad es independiente de la persona que se ejercita con ellos.

\section{CONCLUSIONES}

A modo de conclusión, puede indicarse que los niveles de actividad física de las familias con hijos en Eso son moderados, es más acusada la falta de actividad en los adultos que en los adolescentes. La conciliación es un factor relevante en la práctica de actividad física de los progenitores y en la decisión de quién realiza ejercicio conjuntamente con los hijos. Sin embargo, no se encuentra relación directa de este factor con los niveles de práctica de los hijos (figura 1).
Figura 1. Conciliación y actividad física de familias con hijos adolescentes

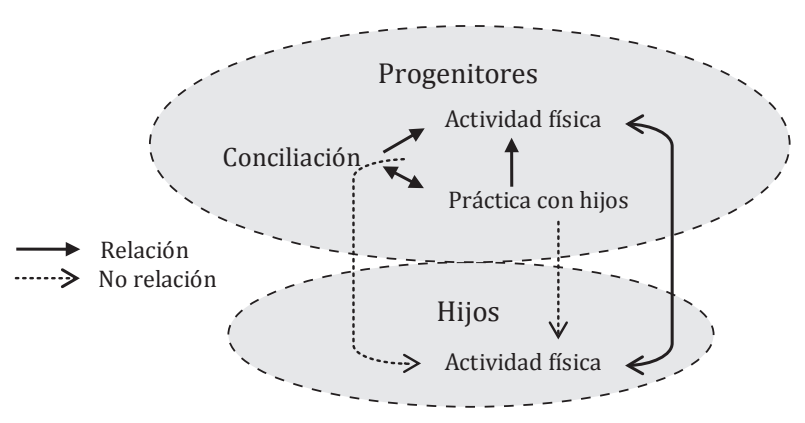

Fuente: Elaboración propia.

Como se aprecia en la figura 1, la conciliación es un factor que afecta prioritariamente a los adultos, sin embargo, puede hablarse de una influencia indirecta sobre los adolescentes, a través del concepto "estilo de vida". En el caso de los estilos de vida activos, las familias cuyos progenitores realizan más actividad física (lo que se ve favorecido por una buena conciliación) transmiten valores, principios y hábitos a sus hijos que contribuyen a que estos sean más activos desde edades muy tempranas.

\section{Limitaciones}

Los niveles de actividad física de los adolescentes han sido informados por los progenitores y no por sus hijos, a pesar de que los adolescentes podrían aportar directamente esta información. Se trata, sin embargo, de una práctica habitual y aceptada en ciencias sociales, principalmente en edades tempranas.

\section{Futuras líneas de investigación}

En futuras investigaciones sería interesante indagar sobre la naturaleza de la actividad física compartida en familia. Generalmente se asume que el ejemplo es lo que facilita y estimula la actividad física de los hijos, pero los datos apuntan a que esta práctica compartida tiene un efecto relevante sobre los niveles de actividad física de los progenitores.

Proponemos ampliar el estudio a otras etapas educativas, como el bachillerato o la enseñanza superior, estudiando la relación existente entre la conciliación de la vida académica y/o laboral, familiar y personal de los jóvenes y sus niveles de actividad física. 


\section{REFERENCIAS}

Beltrán-Carrillo, V. J., Devís-Devís, J., y Peiró-Velert, C. (2012). Actividad física y sedentarismo en adolescentes de la Comunidad Valenciana. Revista Internacional de Medicina y Ciencias de la Actividad Física y el Deporte, 12(45), 122-137.

Borràs, V., Torns, T., y Moreno, S. (2007). Las políticas de conciliación: políticas laborales versus políticas de tiempo. Papers. Revista de Sociologia (83), 83-96.

Canet, L., Grisolía, C., y Querol, R. (2006). Nuevos tiempos, nuevos usos y nuevas tecnologías. Barcelona: Ajuntament de Barcelona. Regidoria Nous Usos Socials del Temps a la Ciutat.

Cecchini, J. A., Méndez, A., y Contreras, O. (2005). Motivos de abandono de la práctica del deporte juvenil. Cuenca: Universidad de Castilla la Mancha.

Codina, N., y Pestana, J. V. (2012). Estudio de la relación del entorno psicosocial en la práctica deportiva de la mujer. Revista de Psicología del Deporte, 21(2), 243251.

Consejo Superior de Deportes (2010). Plan Integral para la Actividad Física y el Deporte. Recuperado de http:// www.planamasd.es/

Crespo, J. M. (2011). Bases para construír una comunicación positiva en la familia. Revista de Investigación en Educación, 9(2), 91-98.

Eurostat (2007). Statistics from the Harmonized European Time Use Survey 2007. Comisión Europea. Recuperado de https://www.h2.scb.se/tus/tus/Disclaimer Menu. html

Fraguela, R., Castiñeiras, J.L.y Varela, L. (2011). Conciliación y actividad física de ocio en familias con hijos en Educación Primaria. Implicaciones para la infancia. Revista de Investigación en Educación, 9(2), 162-173.

García, M. (2006). Posmodernidad y deporte: entre la individualización y la masificación. Encuesta sobre los hábitos deportivos de los españoles 2005. Madrid: CIS y CSD.

García-Moya, I., Moreno, C., Rivera, F., y Jiménez-Iglesias, A. (2012). Iguales, familia y participación en actividades deportivas organizadas durante la adolescencia. Revista de Psicología del Deporte, 21(1), 153-158.

Hicks, J., Basu, K., y Sappey, R. (2011). Controlling the work-life balance?: a case of football non-participation in Australia. International Journal of Sport \& Society, 2(1), 41-53.

Instituto Nacional de Estadística (INE) (2010). Encuesta de empleo del tiempo 2009-2010. Avance de resultados. En Notas de prensa. Instituto Nacional de Estadística. Recuperado de http://www.ine.es/prensa/prensa. html
Isorna, M., Ruiz, F., y Rial, A. (2013). Predictor variables of the dropping out of physical sport activity by teenagers. Cultura, Ciencia y Deporte, 8(23), 93-102.

Kargarfard, M., Kelishadi, R., Ziaee, V., Ardalan, G., Halabchi, F., Mazaheri, R.,... Hayatbakhsh, M. R. (2012). The impact of an after-school physical activity program on health-related fitness of mother/daughter pairs: CASPIAN study. Preventive Medicine, 54(3-4), 219-223.

Martínez, J., Martín, M., Del Hierro, D., Jiménez-Beatty, J. E., y González, M. D. (2008). Barreras a la actividad física en las mujeres adultas y alternativas de conciliación. Actividad Física y Deporte: Ciencia y Profesión, 9, 13-24.

Ministerio de Educación Cultura y Deporte (2012). Estadística de la Educación. Enseñanzas no universitarias. Alumno matriculado. Curso 2011-2012. Resultados detallados. Recuperado de http://www.educacion. gob.es/horizontales/estadisticas /no-universitaria/ alumnado/matriculado/2010-2011-Ultimos-RD.html

Moraleda, I. (2006). Comparecencia ante la Subcomisión del Congreso de los Diputados que analiza la conciliación de la vida laboral, familiar y personal. Madrid: Congreso de los Diputados.

Mulhall, P., Reis, J. y Begum, S. (2011). Early Adolescent Participation in Physical Activity: Correlates With Individual and Family Characteristics. Journal of Physical Activity \& Health, 8(2), 244-252.

Ransdell, L. B., Eastep, E., Taylor, A., Oakland, D., Schmidt, J., Moyer-Mileur, L., y Shultz, B. (2003). Daughters and mothers exercising together (DAMET): Effects of home- and university-based interventions on physical activity behavior and family relations. American Journal of Health Education, 34(1), 19-29.

Root, L. S., y Wooten, L. P. (2008). Time out for family: Shift work, fathers, and sports. Human Resource Management, 47(3), 481-499.

Rueda, S., Artazcoz, L., y Cortés, I. (2009). Usos del tiempo y salud. Barcelona: Ayuntamiento de Barcelona. Sector de Educación, Cultura y Bienestar. Concejalía Usos del Tiempo.

Salazar, C. M., Feu, S., Vizuete Carrisoza, M., y De la CruzSánchez, E. (2013). Social and residential context as determinants of physical activity patterns of students at the university of Colima. Cultura, Ciencia y Deporte, 8(23), 103-112.

Tobío, C., y Fernández, J. A. (1999). Monoparentalidad, trabajo y familia. Revista Internacional de Sociología (RIS), 22, 67-97. 
Valdemoros, Mạ . A., Ponce de León, A., Ramos, R., y Sanz, E. (2011). Pedagogía de la convivencia y educación no formal: un estudio desde el ocio físico-deportivo, los valores y la familia. European Journal of Education and Psychology, 4(1), 33-49.
Wagner, A., Klein-Platat, C., Arveiler, D., Haan, M. C., Schlienger, J., y Simon, C. (2004). Parent-child physical activity relationships in 12-year old French students do not depend on family socioeconomic status. Diabetes \& Metabolism, 30(4), 359-366. 
